\title{
Detecting with PMUs the onset of voltage instability caused by a large disturbance
}

\author{
Mevludin Glavic, Senior Member, IEEE
}

Thierry Van Cutsem, Fellow, IEEE

\begin{abstract}
This paper deals with long-term voltage instability triggered by the outage of transmission or generation equipments. It is shown how the onset of voltage instability could be detected in real-time from post-disturbance bus voltages provided by Phasor Measurement Units (PMUs), assuming observability of the whole region prone to voltage instability. An extended set of equilibrium equations is fitted to the measurements and the change in sign of sensitivities is monitored. The latter are easily computed even for a large system. The important effects of overexcitation limiters and load tap changers are taken into account. The approach is illustrated on a 52-bus 20-machine system, where PMU outputs are simulated from detailed time simulation.
\end{abstract}

Index Terms - Long-term voltage stability, instability detection, phasor measurement units, sensitivity analysis, wide-area monitoring

\section{INTRODUCTION}

Voltage instability is known to be a potential source of power system blackouts [1], [2]. As economic and environmental considerations will force system operation close to stability limits, the need for effective means to detect an approaching critical condition, as well as to counteract a developing instability is becoming more important [3].

Recent technological advances in terms of computational facilities, networking infrastructure, communications, and phasor measurements with time synchronizing capabilities, have created new opportunities for designing wide-area monitoring, control and protection schemes [3]. These advances open new perspectives in various areas from mere monitoring to System Protection Schemes (SPS) [3], [4].

\section{A. On the use of PMUs for voltage instability detection}

This includes the possibility to detect and control an impeding voltage instability. This paper is devoted to the detection of long-term voltage instability, driven typically by Load Tap Changers (LTCs), OverExcitation Limiters (OELs) and restorative loads. These long-term dynamics can be monitored through PMUs [5], properly located in the network [6], and able to provide successive system "snapshots" at a much higher rate than the present-day SCADA systems. Although the PMU accuracy is limited by that of current and potential transformers, transducers, etc. their main advantage lies in the availability of well synchronized data.

M. Glavic (glavic@montefiore.ulg.ac.be) is a visiting researcher at the Dept. of Electrical Engineering and Computer Science of the University of Liège, Sart Tilman B37, B-4000 Liège, Belgium.

T. Van Cutsem (t.vancutsem@ulg.ac.be) is a research director of the FRS (Fund for Scientific Research-FNRS) and adjunct professor at the same department.
There are two complementary lines of defence against voltage instability [1], [2]: (i) preventively: check system security margins with respect to "credible" (typically N-1) events; (ii) correctively: face more severe disturbances leading to emergency conditions through SPS. As regards preventive aspects, mature techniques are now available to perform Voltage Security Assessment (VSA). Simulation techniques are available to answer "what if" questions and compute load power margins, for instance. PMUs can help in this respect by improving the quality of state estimation, i.e. by providing better initial operating points for VSA applications. However, we believe that the second aspect is where PMUs could contribute more significantly, and complement if not replace the existing schemes based on undervoltage measurements.

Long-term voltage instability can be triggered by the outage of transmission or generation equipments or unacceptable load increase. Most of the incidents experienced so far were triggered by the former category of events, and relatively fewer by load increases. Furthermore, the latter leave (properly trained) operators some time to react and can be anticipated by standard VSA tools computing loadability limits [2]. Hence, PMUs appear to be most useful in dealing with large-disturbance voltage instability, where operators are left with no or very little time to react. This work focuses on large-disturbance scenarios, which have been comparatively less investigated in the literature.

Voltage stability monitoring approaches can be classified into two broad categories: (i) methods based on local measurements, which can be implemented in a distributed manner, i.e. at several locations throughout the system with few or no information exchange between these locations; (ii) those requiring observability of the whole region prone to voltage instability. In principle, the former do not require time synchronization of the PMUs, while the latter should preferably rely on a state estimator filtering the data provided by PMUs. The model of this estimator is linear if only PMUs are involved [6], [7].

\section{B. Methods based on local measurements}

Most of the methods based on local measurements rely on the Thévenin impedance matching condition [8] or its extensions [9]-[13].

In the method proposed in [8] the measurements collected at one load bus are used to obtain the Thévenin equivalent of the system seen from this bus, as well as the apparent impedance of the load. The parameters of the Thévenin equivalent are estimated using a least-square method once two or more sets of measurements are available. The same concept is used in 
[9] but proximity to instability was expressed in terms of power margin, while a feasibility study of implementation into voltage security monitoring and control tools was reported. The extension of this scheme described in [10] include measurements taken at remote buses to assist the estimation of the Thévenin equivalent. To avoid the time delay of leastsquare estimation, it is proposed in [11] to obtain the Thévenin equivalent from measurements taken at a single time but at both ends of a transmission corridor. A two-bus equivalent is also used in [13] where the large network behind a load bus is represented by a source in series with a transmission line (using time synchronized PMUs), preserving power flow and voltage information at the bus of concern.

Application of Thévenin equivalent and impedance matching faces some difficulties. The first one, of theoretical nature, has to do with the adequacy of observing a single load when maximum power is reached over a set of loads. In this respect, it has been shown in [14] that the impedance matching condition is necessarily met after meeting the maximum load condition that is reflected into singularity of the corresponding Jacobian. Next, the Thévenin impedance has to be estimated from measurements gathered over a time window that should be wide enough for the operating conditions to change, but narrow enough to satisfy the constant Thévenin impedance assumption [11]. It is not clear whether the technique is robust enough to be applied over the time interval that follows a severe disturbance (instead of during a smooth load increase).

A step towards wide-area monitoring was made in the method of [15] which relies a wide-area network of PMUs and uses a variant of the impedance matching condition together with estimates of generator reactive power reserves to identify critical operating conditions. This reference also extends the impedance matching condition by including the non-impedance part of loads into the Thévenin equivalent.

\section{Methods assuming observability of the whole voltage un- stable region}

Being able, in some future, to make a whole region prone to voltage instability observable through PMUs definitely opens new perspectives.

In principle, several among the formerly proposed voltage stability indices [16] are suitable. Some of these indices, such as the sensitivities considered in this paper, are not considered appropriate for preventive VSA purposes, where power margins are preferred as a security index easily interpreted by operators. They are, however, suitable for emergency condition detection, as demonstrated in this paper.

Among the recently proposed indices, Ref. [17] approximates the voltage drop across the Thévenin impedance by the sum of the absolute values of the complex voltage drops over the branches located on the shortest path from the load bus of concern to a generator under voltage control. The impedance matching criterion is then replaced by a voltage drop matching criterion. Some tuning of the alarm threshold is needed to compensate for the heuristic nature of the method.

An accurate identification of voltage instability inception can be obtained from singularity conditions of a properly computed Jacobian matrix. Coupling between time simulation and eigenvalue analysis of Jacobians have been proposed more than a decade ago, either to validate eigenvalue analysis derived from power flow equations [18] or to identify the most effective control actions in a post-contingency voltage unstable scenario [19]. More recently sensitivity techniques were revisited and extended in [20]. In this paper, we basically propose to replace the above off-line noise-free computations by fast ones performed in real-time and based on the outputs of PMUs.

In the same spirit, Ref. [21] proposed a technique based on PMUs and using the Jacobian of the standard power flow equations. Beside the fact that the latter carry some approximation [2], only smooth load increase scenarios were considered.

\section{Objectives of this work}

The approach reported in this paper belongs to the second category of methods. We simulate plausible raw PMU or state estimator outputs by adding noise to the bus voltages provided by snapshots of detailed time simulation. To each so obtained snapshot we fit an extended set of equilibrium equations from which we perform efficient sensitivity analysis, taking into account the important effects of OELs and LTCs. As already mentioned, we focus on the more interesting (and challenging) problem of identifying the onset of voltage instability triggered by a large disturbance. Last but not least, we incorporate filtering to deal with the inevitable noise stemming from measuring devices as well as fast transients.

This work does not consider the problem of PMU placement, availability of communications infrastructure, and if/how the system state is provided by a linear state estimator [6]. Instead, we simply assume that a network of PMUs exists, together with adequate communication, and that system state is known through bus voltage phasors all relative to a common reference. We also assume that the network of PMUs is able to monitor system topology in real-time [22]. The transmission of OEL activation information is also discussed.

\section{THE PROPOSED APPROACH}

\section{A. Modelling and sensitivities}

In stability studies, the general dynamic model of a power system takes on the form:

$$
\begin{aligned}
\mathbf{0} & =\mathbf{g}\left(\mathbf{x}, \mathbf{y}, \mathbf{z}_{c}, \mathbf{z}_{d}\right) \\
\dot{\mathbf{x}} & =\mathbf{f}\left(\mathbf{x}, \mathbf{y}, \mathbf{z}_{c}, \mathbf{z}_{d}\right) \\
\dot{\mathbf{z}}_{\mathbf{c}} & =\mathbf{h}_{c}\left(\mathbf{x}, \mathbf{y}, \mathbf{z}_{c}, \mathbf{z}_{d}\right) \\
\mathbf{z}_{d}\left(t_{k}^{+}\right) & =\mathbf{h}_{d}\left(\mathbf{x}, \mathbf{y}, \mathbf{z}_{c}, \mathbf{z}_{d}\left(t_{k}^{-}\right)\right)
\end{aligned}
$$

The algebraic equations (1) relate to the network, considered to respond infinitely fast. They involve the vector $\mathbf{y}$ of bus voltages.

The differential equations (2) and the corresponding state vector $\mathrm{x}$ relate to the short-term dynamics of generators, turbines, governors, Automatic Voltage Regulators (AVRs), static var compensators, induction motors, HVDC links, etc. 
The differential equations (3) and corresponding state vector $\mathbf{z}_{c}$ capture the continuous long-term dynamics of secondary frequency and voltage control, load self-restoration, etc.

Finally, the discrete-time equations (4) capture discrete events that stem from: (i) controllers acting with various delays on shunt compensation, generator set points, LTCs, etc., (ii) equipment protections such as OELs, and (iii) possibly, system protection schemes acting on loads and/or generators. The corresponding vector $\mathbf{z}_{d}$ includes shunt susceptances, transformer ratios, etc. which undergo step changes from $\mathbf{z}_{d}\left(t_{k}^{-}\right)$to $\mathbf{z}_{d}\left(t_{k}^{+}\right)$ at some times $t_{k}$ dictated by the whole system dynamics.

For stability analysis we consider a single long-term state vector $\mathbf{z}$ and formally replace $(3,4)$ by the single continuoustime equation:

$$
\dot{\mathbf{z}}=\mathbf{h}(\mathbf{x}, \mathbf{y}, \mathbf{z})
$$

without loss of generality, for reasons that will appear in the sequel. A linearization of $(1,2,5)$ gives:

$$
\left[\begin{array}{c}
\mathbf{0} \\
\Delta \dot{\mathbf{x}} \\
\Delta \dot{\mathbf{z}}
\end{array}\right]=\underbrace{\left[\begin{array}{ccc}
\mathrm{g}_{\mathrm{y}} & \mathrm{g}_{\mathrm{x}} & \mathrm{g}_{\mathrm{z}} \\
\mathrm{f}_{\mathrm{y}} & \mathbf{f}_{\mathrm{x}} & \mathbf{f}_{\mathrm{z}} \\
\mathbf{h}_{\mathbf{y}} & \mathbf{h}_{\mathbf{x}} & \mathbf{h}_{\mathrm{z}}
\end{array}\right]}_{\mathbf{J}}\left[\begin{array}{c}
\Delta \mathbf{y} \\
\Delta \mathbf{x} \\
\Delta \mathbf{z}
\end{array}\right]
$$

where $\mathbf{g}_{\mathbf{y}}$ denotes the Jacobian matrix of $\mathbf{g}$ with respect to $\mathbf{y}$, and similarly for the other sub-matrices. One easily obtains the linearized state space model:

$$
\begin{aligned}
& {\left[\begin{array}{c}
\Delta \dot{\mathbf{x}} \\
\Delta \dot{\mathbf{z}}
\end{array}\right]=\mathbf{A}\left[\begin{array}{c}
\Delta \mathbf{x} \\
\Delta \mathbf{z}
\end{array}\right] } \\
& \text { with } \mathbf{A}=\left[\begin{array}{cc}
\mathbf{f}_{\mathbf{x}} & \mathbf{f}_{\mathbf{z}} \\
\mathbf{h}_{\mathbf{x}} & \mathbf{h}_{\mathbf{z}}
\end{array}\right]-\left[\begin{array}{c}
\mathbf{f}_{\mathbf{y}} \\
\mathbf{h}_{\mathbf{y}}
\end{array}\right] \mathbf{g}_{\mathbf{y}}{ }^{-1}\left[\begin{array}{ll}
\mathbf{g}_{\mathbf{x}} & \mathbf{g}_{\mathbf{z}}
\end{array}\right](8)
\end{aligned}
$$

When a system undergoes long-term voltage instability, one real eigenvalue of the state matrix $\mathbf{A}$ goes from negative to positive. Using Schur's formula, it is easily shown that the same happens to one real eigenvalue of the unreduced Jacobian $\mathbf{J}$ involved in (6) [2].

Note that $\mathbf{J}$ is nothing but the Jacobian matrix of the longterm equilibrium equations:

$$
\begin{aligned}
& \mathbf{0}=\mathbf{g}(\mathbf{x}, \mathbf{y}, \mathbf{z}) \\
& \mathbf{0}=\mathbf{f}(\mathbf{x}, \mathbf{y}, \mathbf{z}) \\
& \mathbf{0}=\mathbf{h}(\mathbf{x}, \mathbf{y}, \mathbf{z})
\end{aligned}
$$

To detect that one real eigenvalue of $\mathbf{J}$ crossed zero, there is no need to compute eigenvalues of $\mathbf{J}$; sensitivities obtained from $\mathbf{J}^{-1}$ can be used instead [2], [20].

We consider the sensitivities $S_{Q_{g} \mathbf{Q}_{l}}$ of the total reactive power generation $Q_{g}$ to individual load reactive powers $\mathbf{Q}_{l}$. As they involve the reactive power generation of all generators and compensators, they provide a global monitoring of the system [2], [20]. They are easily obtained from a general sensitivity formula as:

$$
S_{Q_{g} \mathbf{Q}_{l}}=-\left[\begin{array}{lll}
\mathbf{g}_{\mathbf{Q}_{l}}^{T} & \mathbf{f}_{\mathbf{Q}_{l}}^{T} & \mathbf{h}_{\mathbf{Q}_{l}}^{T}
\end{array}\right]\left[\mathbf{J}^{T}\right]^{-1}\left[\begin{array}{c}
\nabla_{\mathbf{y}} Q_{g} \\
\nabla_{\mathbf{x}} Q_{g} \\
\nabla_{\mathbf{z}} Q_{g}
\end{array}\right]
$$

where $\nabla_{\mathbf{y}} Q_{g}$ denotes the gradient of $Q_{g}$ with respect to $\mathbf{y}$, and similarly for the other sub-vectors. Since they involve the inverse of the Jacobian matrix, the sensitivities change sign passing (theoretically) through infinity when one real eigenvalue changes from negative to positive.

The computation of $S_{Q_{g} \mathbf{Q}_{l}}$ from (12) merely requires to solve one linear system with the sparse matrix $\mathbf{J}$ of coefficients. The other vectors and matrices involved in (12) are simple and also very sparse. There is thus no problem computing the above sensitivities at high a rate, even for a large system.

\section{B. Deriving practical long-term equilibrium equations}

As indicated previously, the method requires to consider a set of long-term equilibrium equations from which the Jacobian is computed and factorized.

1) Network: We consider a standard representation based on the nodal admittance matrix $\overline{\mathbf{Y}}$ and written in rectangular coordinates. The current-voltage relations $\overline{\mathbf{I}}=\overline{\mathbf{Y}} \overline{\mathbf{V}}$ are decomposed into real and imaginary parts:

$$
\begin{aligned}
& \mathbf{G} \mathbf{v}_{x}-\mathbf{B} \mathbf{v}_{y}-\mathbf{i}_{x}=\mathbf{0} \\
& \mathbf{B} \mathbf{v}_{x}+\mathbf{G v}_{y}-\mathbf{i}_{y}=\mathbf{0}
\end{aligned}
$$

where $\overline{\mathbf{Y}}=\mathbf{G}+j \mathbf{B}, \overline{\mathbf{V}}=\mathbf{v}_{x}+j \mathbf{v}_{y}$ and $\overline{\mathbf{I}}=\mathbf{i}_{x}+j \mathbf{i}_{y}$.

We take one of the buses as reference by setting the phase angle of its voltage to zero, or equivalently:

$$
v_{y r}=0
$$

where $r$ is the number of the bus of concern.

2) Short-term dynamics at equilibrium: Formally, the equations (10) are obtained from the equilibrium (i.e. steady-state) conditions of a detailed short-term dynamics model. In practice, however, the steady-state model of a generator equipped with AVR, turbine and speed governor can be replaced by the small set of equations detailed hereafter. Other components can be handled similarly.

The synchronous machine in steady state is described by the well-known Park equations in $(d, q)$ axes:

$$
\left(\begin{array}{c}
0 \\
E_{q}^{s}
\end{array}\right)=\left(\begin{array}{c}
v_{d} \\
v_{q}
\end{array}\right)+\left(\begin{array}{cc}
0 & X_{q}^{s} \\
-X_{d}^{s} & 0
\end{array}\right)\left(\begin{array}{c}
i_{d} \\
i_{q}
\end{array}\right)
$$

where the armature resistance has been neglected, $E_{q}^{s}$ is the emf behind saturated synchronous reactances, and $X_{d}^{s}$ and $X_{q}^{s}$ are the saturated direct- and quadrature-axis synchronous reactances, respectively. When referring the voltage and current to the network reference, the above equation becomes:

$$
\begin{aligned}
& \left(\begin{array}{c}
0 \\
E_{q}^{s}
\end{array}\right)=\left(\begin{array}{cc}
-\sin \delta & \cos \delta \\
\cos \delta & \sin \delta
\end{array}\right)\left(\begin{array}{c}
v_{x} \\
v_{y}
\end{array}\right) \\
& +\left(\begin{array}{cc}
0 & X_{q}^{s} \\
-X_{d}^{s} & 0
\end{array}\right)\left(\begin{array}{cc}
-\sin \delta & \cos \delta \\
\cos \delta & \sin \delta
\end{array}\right)\left(\begin{array}{l}
i_{x} \\
i_{y}
\end{array}\right)
\end{aligned}
$$

where $\delta$ is the rotor angle, i.e. the angle between the $q$ axis and the network reference, as shown in Fig. 1.

The saturation is accounted for by:

$$
E_{q}=\left(1+m\left(V_{\ell}\right)^{n}\right) E_{q}^{s}
$$

where $E_{q}$ is the emf proportional to field current, and $V_{\ell}$ is the voltage behind the leakage reactance $X_{\ell}$ given by:

$$
V_{\ell}=\left|\bar{V}+j X_{\ell} \bar{I}\right|=\left[\left(v_{x}-X_{\ell} i_{y}\right)^{2}+\left(v_{y}+X_{\ell} i_{x}\right)^{2}\right]^{1 / 2}
$$




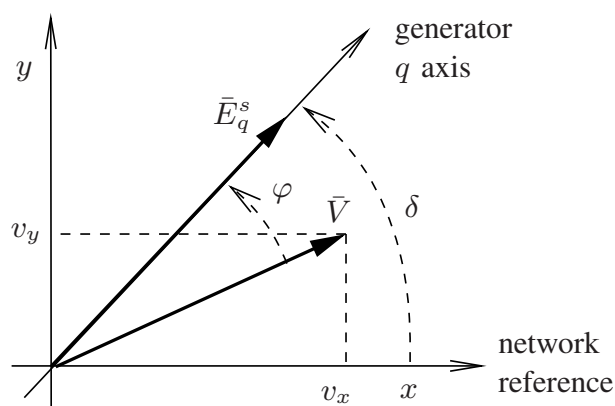

Fig. 1. Phasor diagram and reference definition

The reactances $X_{d}^{s}$ and $X_{q}^{s}$ relate to their unsaturated values $X_{d}$ and $X_{q}$ through:

$$
X_{d}^{s}=X_{\ell}+\frac{X_{d}-X_{\ell}}{1+m\left(V_{l}\right)^{n}} \quad X_{q}^{s}=X_{\ell}+\frac{X_{q}-X_{\ell}}{1+m\left(V_{l}\right)^{n}}
$$

The voltage regulation is accounted for by:

$$
E_{q}=G\left(V^{o}-V\right) \Leftrightarrow E_{q}-G\left(V^{o}-\sqrt{v_{x}^{2}+v_{y}^{2}}\right)=0
$$

if the generator is under control of an AVR with open-loop static gain $G$, and by:

$$
E_{q}=E_{q}^{l i m}
$$

is the machine is under field current limit.

The frequency regulation is accounted for by:

$$
P=P^{o}-\alpha \Delta f_{s} \Leftrightarrow v_{x} i_{x}+v_{y} i_{y}-P^{o}+\alpha \Delta f_{s}=0
$$

where $\alpha$ is a participation factor involving the permanent speed droop, and $\Delta f_{s}$ is the deviation of the system frequency with respect to its nominal value.

Replacing $X_{d}^{s}$ and $X_{q}^{s}$ by (20) and $V_{\ell}$ by (19), the five equations $(17,18,21$ or 22,23$)$ make up the set of shortequilibrium equations (10) relative to each generator. They involve five $\mathbf{x}$ variables, namely $i_{x}, i_{y}, \delta, E_{q}$ and $E_{q}^{s}$. Note that, under the assumption of uniform frequency, the variable $\Delta f_{s}$ is common to all generators and balanced by Eq. (15).

3) Long-term dynamics at equilibrium: As indicated by (11) loads must be modeled according to their long-term behaviour. LTCs controlling load voltages play an important role in long-term voltage stability. Neglecting deadband and LTC limits, the equilibrium equation (11) of an LTC is merely:

$$
V=V^{o}
$$

where $V$ is the controlled load voltage and $V^{o}$ the corresponding setpoint. As an alternative to including equations (24), the loads can be represented as constant power (since restoring load voltage is equivalent to restoring load powers), which leads to the following equations at each LTC-controlled bus:

$$
\begin{aligned}
& v_{x} i_{x}+v_{y} i_{y}+P_{o}=0 \\
& v_{y} i_{x}-v_{x} i_{y}+Q_{o}=0
\end{aligned}
$$

\section{Matching equations and variables to real-time snapshots}

As previously explained, at each time step, synchronized values of the bus voltages phasors $\hat{\mathbf{V}}$ are assumed to be received either from PMUs or (preferably) from a state estimator. Taking the voltage at the $r$-th bus as reference, and decomposing $\hat{\mathbf{V}}$ into real and imaginary parts yields the vectors $\hat{\mathbf{v}}_{\mathbf{x}}$ and $\hat{\mathbf{v}}_{\mathbf{y}}$. From the network equations $(13,14)$ the nodal current components $\hat{\mathbf{i}}_{\mathbf{x}}$ and $\hat{\mathbf{i}}_{\mathbf{y}}$ are easily obtained.

As the short-term dynamics are not tracked in the proposed method, the short-term variables $\mathrm{x}$ are initialized assuming these dynamics to be at equilibrium at any time, i.e. using (10) or equivalent relations. We detail hereafter the procedure for the synchronous generator.

First, the active and reactive current are obtained from:

$$
\begin{aligned}
& I_{P}=P / V=\left(v_{x} i_{x}+v_{y} i_{y}\right) / \sqrt{v_{x}^{2}+v_{y}^{2}} \\
& I_{Q}=Q / V=\left(v_{y} i_{x}-v_{x} i_{y}\right) / \sqrt{v_{x}^{2}+v_{y}^{2}}
\end{aligned}
$$

while the voltage $V_{\ell}$ is computed from (19), from which the saturated reactances can be determined using (20). Next, the internal (or load) angle $\varphi$ (see Fig. 1) is determined [2]:

$$
\varphi=\operatorname{arctg}\left(\frac{X_{q}^{s} I_{P}}{V+X_{q}^{s} I_{Q}}\right)
$$

as well as the rotor angle (see same figure):

$$
\delta=\varphi+\operatorname{arctg}\left(v_{y} / v_{x}\right)
$$

Finally the emf $E_{q}^{s}$ is obtained from:

$$
E_{q}^{s}=\left(V+X_{d}^{s} I_{Q}\right) \cos \varphi+X_{d}^{s} I_{P} \sin \varphi
$$

and the emf $E_{q}$ from (18).

It is essential to account for OEL activation. To avoid sending the status of OELs, $E_{q}$ can be compared to $E_{q}^{\text {lim }}$ corresponding to the rotor current enforced by the OEL. If $E_{q}<E_{q}^{l i m}$, the AVR is active and (21) is used. If $E_{q}>E_{q}^{l i m}$ for some time (accounting for temporary excitation peaks after a large disturbance, although this can be mitigated through filtering), it is concluded that the machine is overexcited and the OEL is going act after some delay. In order to anticipate the future OEL activation, (22) is used to build the Jacobian. This remains in effect as long as $E_{q}$ lies in an interval $\left[E_{q}^{\lim }-\epsilon E_{q}^{\text {lim }}+\epsilon\right]$, where $\epsilon$ accounts for noise, transients and OEL inaccuracy.

\section{Filtering}

Filtering is required to attenuate the effect of measurement noises and system transients. Preprocessing by a state estimator will somewhat reduce the noise effects but some filtering is still needed, also to smoothen the effect of transients, particularly electromechanical oscillations, that are not taken into account in the model.

In this work, a simple Moving Average (MA) filter has been considered. This consists of replacing the incoming data with the average of a number of past values of the same quantity. Thus, at discrete time $k$ we consider:

$$
\bar{z}(k)=\frac{z(k)+z(k-1)+\ldots+z(k-N+1)}{N}
$$

where $N$ is the size of the moving window. The signals to be filtered are discussed in the next section. 


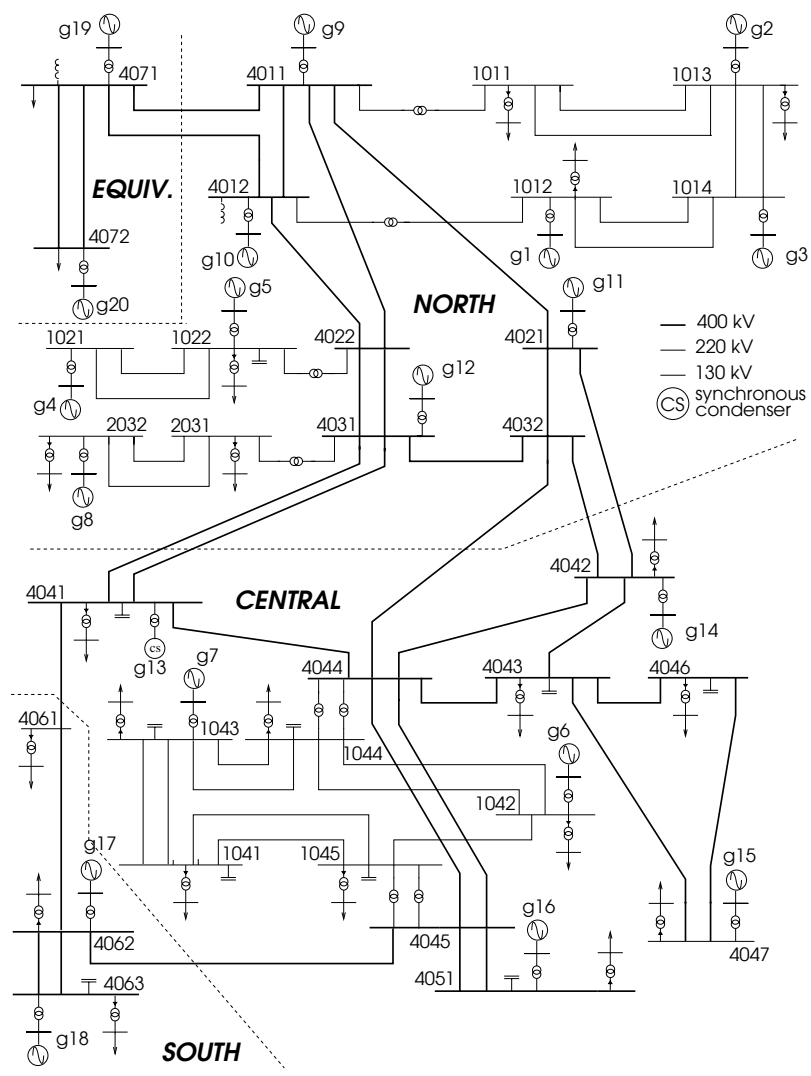

Fig. 2. Nordic-32 test system

\section{Simulation RESUlts}

\section{A. Nordic-32 test system}

We report on results obtained with a slightly modified version of the so-called Nordic-32 test system, detailed in [23]. The one-line diagram of this 52-bus, 20-machine system is shown in Fig. 2.

Each generator is represented with 3 or 4 rotor windings (sub-transient time constants are thus taken into account), a simple governor (for generators in the North and Equiv areas; the other ones do not participate in frequency control) and a simple AVR including an OEL.

Each load is modelled as constant current for the active power and constant admittance for the reactive power. In addition, each load is fed through a transformer with LTC. There is a delay of $30 \mathrm{~s}$ on the first tap change and a shorter delay on the subsequent steps.

The "measured" voltage phasors $\hat{\mathbf{V}}$ are obtained by sampling the values of $\mathbf{v}_{x}$ and $\mathbf{v}_{y}$ provided by the time simulation. We assume that a snapshot is taken and processed every $100 \mathrm{~ms}$. Communications delays are not considered, as they are not significant for the dynamics of concern.

\section{B. Disturbance and system evolution}

The disturbance considered in the sequel is a three-phase short circuit applied at $t=0.95 \mathrm{~s}$ near bus 4044 and cleared after $100 \mathrm{~ms}$ by opening the faulted line 4032-4044.

The system initial operating point is insecure, due to low reactive power reserves on some generators and too a large a power transfer from North to Central areas. As a result, the

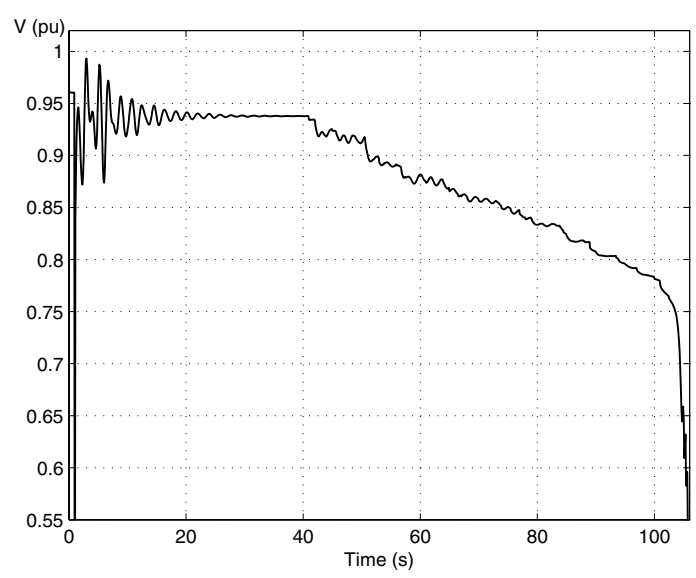

Fig. 3. Voltage at bus 1041

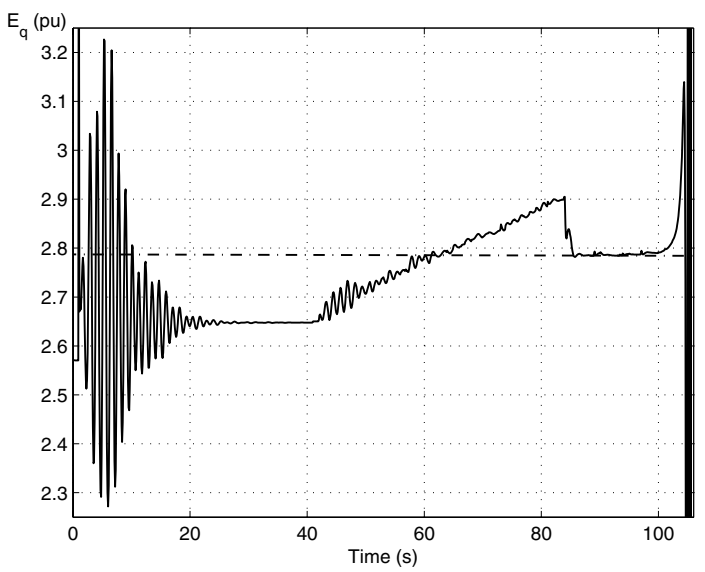

Fig. 4. $E_{q}$ emf of generator g6 computed from measurements

disturbance makes the system long-term voltage unstable, as can be seen from the evolution of voltage at bus 1041 in Fig. 3.

The system evolves over some 105 seconds under the effect of LTCs trying unsuccessfully to restore distribution voltages (and hence load powers) and OELs acting on various generators (namely on g5 at $t=30.8 \mathrm{~s}, \mathrm{~g} 14$ at $t=43.1 \mathrm{~s}$, $\mathrm{g} 15$ at $t=46.4 \mathrm{~s}, \mathrm{~g} 7$ at $t=51.6 \mathrm{~s}, \mathrm{~g} 12$ at $t=51.7 \mathrm{~s}$, g16 at $t=57.8 \mathrm{~s}$ and eventually g6 at $t=85.2 \mathrm{~s}$ ), thereby further reducing the maximum power that can be transmitted to loads [2]. Eventually, long-term voltage instability results in a loss of short-term stability in the form of field current limited generators loosing synchronism.

\section{Monitoring of generator limitation}

The OEL of generator g6 is activated at $t=85.2 \mathrm{~s}$. The evolution of the $E_{q}$ emf of that generator, calculated each 100 $\mathrm{ms}$ from the voltage phasors, is shown in Fig. 4, where the dash-dotted horizontal line corresponds to the OEL limit. This limit is crossed for short, non significant periods of time during the electromechanical transients that follow the short-circuit. On the other hand, it is easily seen that for $t \in\left[\begin{array}{ll}63 & 85\end{array}\right] \mathrm{s}$, the rotor current is above its limit, and for $t>85 \mathrm{~s}$, the OEL has acted. This is inferred without receiving information from the OEL itself. The final transients correspond to loss of synchronism. 


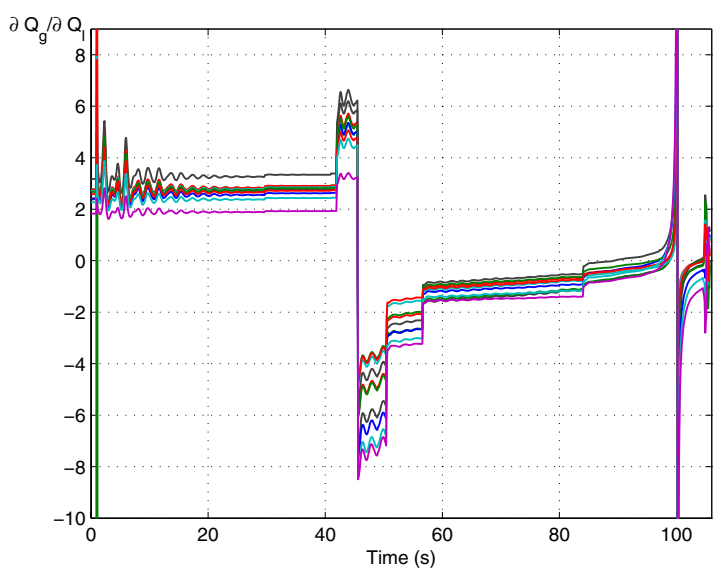

Fig. 5. Sensitivities $S_{Q_{g} \mathbf{Q}_{1}}$ at buses of Central area; no measurement noise

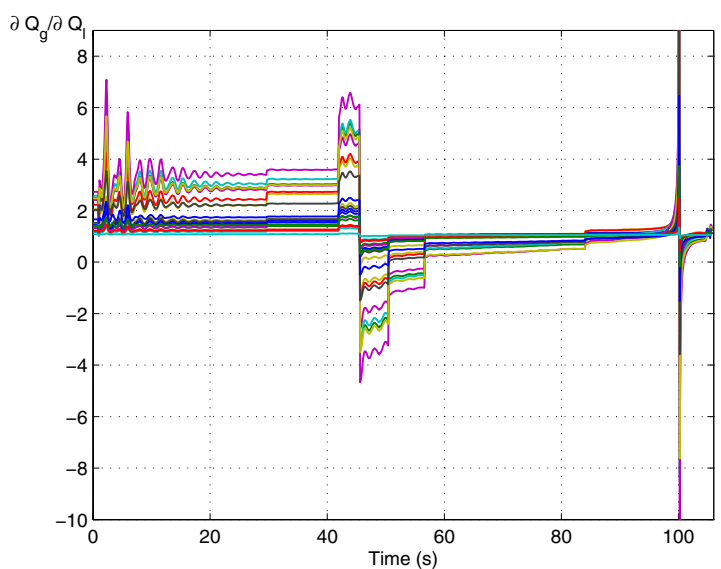

Fig. 6. Sensitivities $S_{Q_{g} \mathbf{Q}_{\mathbf{1}}}$ at buses of North area; no measurement noise

\section{Sensitivities obtained from exact measurements}

The evolution of the sensitivities $S_{Q_{g} \mathbf{Q}_{1}}$ directly computed from the voltage phasors $\hat{\mathbf{V}}$ is shown in Figs. 5 and 6, for the Central and North areas, respectively. A typical value before disturbance is 2, at most 4 (pu/pu or Mvar/Mvar). As can be seen, the sensitivities change sign while passing through much larger values at $t=45.7 \mathrm{~s}$. The long-term instability is thus detected $44.7 \mathrm{~s}$ after the fault is cleared and some $60 \mathrm{~s}$ before the system collapses. At $t=45.7 \mathrm{~s}$ the lowest transmission voltage, recorded at bus 1041, is around 0.92 pu (see Fig. 3). Thus, an early detection of impeding instability is obtained.

In principle all sensitivities should exhibit huge (in theory infinite) values just before and just after changing sign. However, in the presence of generator limits, they may just undergo a jump from a large positive to a large negative values. The corresponding instability mechanism and its impact on sensitivities were analyzed in [24], [2].

All sensitivities, except a few ones relative to North area, change sign. However the change in sign is less pronounced in the North area, where sensitivities come back to positive after some $10 \mathrm{~s}$, while those relative to Central area remain negative. This behaviour is further analyzed in [20]. Thus, a ranking of the sensitivities clearly identifies the area in trouble.

The other change in sign through large values occurring near $t=100 \mathrm{~s}$ is probably caused by another zero crossing eigenvalue. In any case, voltages are so depressed when this

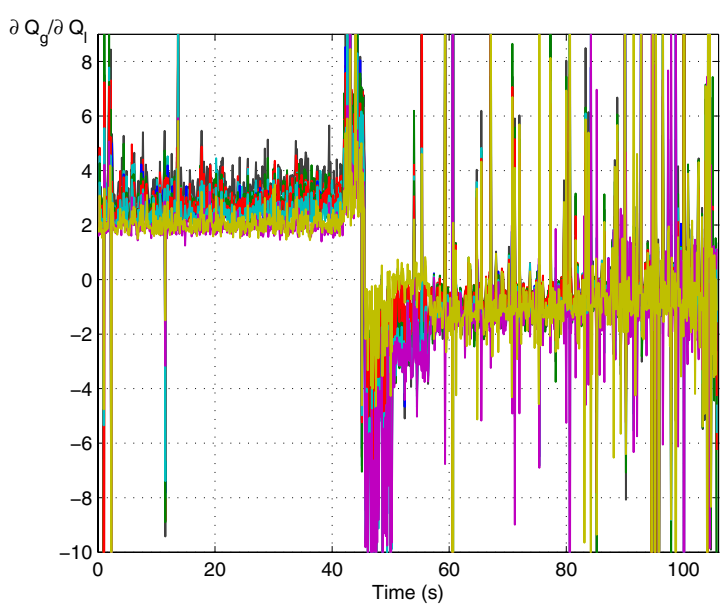

Fig. 7. Sensitivities $S_{Q_{g} \mathbf{Q}_{1}}$ obtained from noisy measurements

takes place that further investigations are of theoretical interest essentially.

\section{E. Sensitivities obtained from noisy measurements}

Peaks during the fault-on conditions as well as oscillations due to unmodeled dynamics can be observed in Figs. 5 and 6. They do not prevent instability detection.

We now consider the effect of inaccuracies affecting the phasors $\hat{\mathbf{V}}$. To this purpose a random noise has been added to each component of $\mathbf{v}_{x}$ and $\mathbf{v}_{y}$. For simplicity, the random variables added to the two components of the same voltage phasor have been assumed uncorrelated. Each random variable is normally distributed with zero mean and standard deviation $\sigma=0.04 \mathrm{pu}$. This high noise was intentionally chosen to test the method in drastic conditions that could result from rather large measurement errors.

The sensitivities directly computed from the noisy voltage phasors $\hat{\mathbf{V}}$ are shown in Fig. 7 for the Central area only. Although one can still guess the change in sign, the huge number of peaks hinders any reliable diagnostic.

Hence, the MA filter of (32) has been used. A window size of 20 points (corresponding to a time window of $1.9 \mathrm{~s}$ ) has been considered, except for the first 19 points where the window size is equal to the number of available snapshots.

Two options have been considered.

In the first one, sensitivities are computed from the incoming measurements and the filter is applied to the successive sensitivity values. The corresponding results are shown in Fig. 8. Although filtering is already effective, some spurious oscillations around zero still make a diagnosis a bit difficult.

The second option consists of applying the MA filter to the incoming voltage phasor $\hat{\mathbf{V}}$ and using the so filtered values to compute the sensitivities. The corresponding results are given in Fig. 9. Much better results are obtained, and the clear picture that was obtained in the absence of noise is restituted. Furthermore, the oscillations caused by the fault and observed in Fig. 5 have been significantly eliminated.

The better performance can be explained as follows. Most likely, the Jacobian used to compute the sensitivities is not well conditioned, especially in the neighborhood of the point 


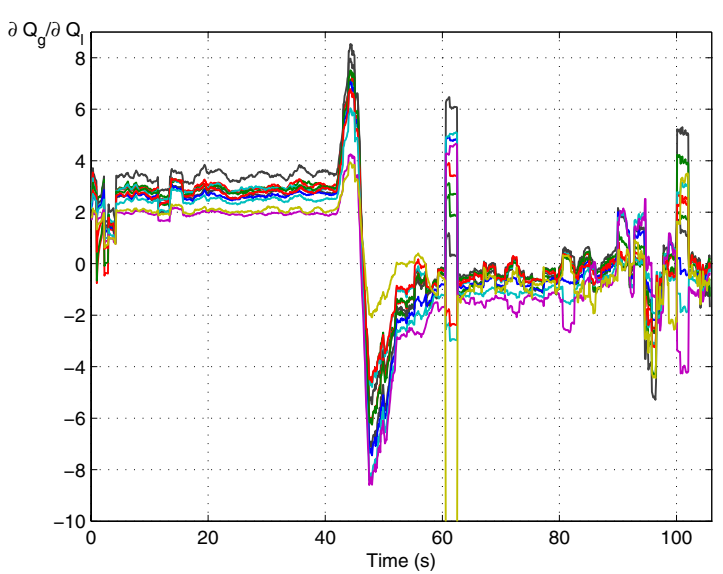

Fig. 8. Sensitivities $S_{Q_{g} \mathbf{Q}_{\mathbf{1}}}$ computed from noisy measurements, then filtered

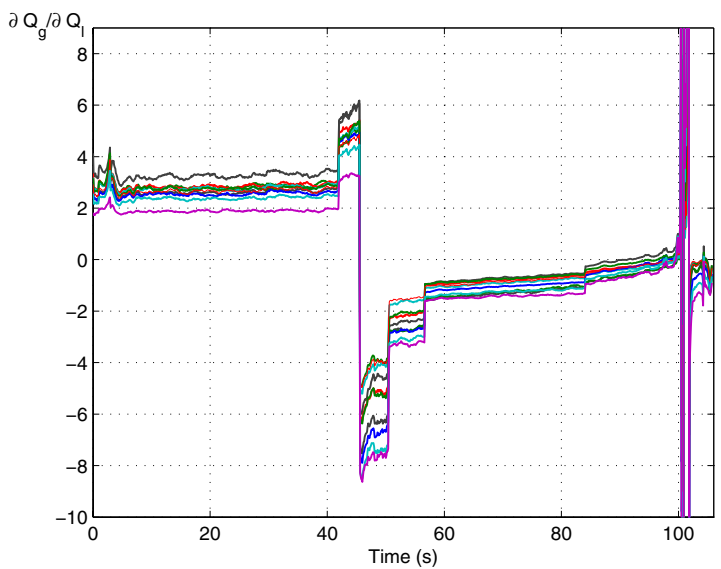

Fig. 9. Sensitivities $S_{Q_{g} \mathbf{Q}_{1}}$ computed from previously filtered measurements

where one eigenvalue crosses zero. This amplifies the effect of the random components of voltages. The latter are less present in the average voltage values provided by the MA filter.

It is thus concluded that the MA filter should be applied to the input voltages, even more if one relies directly on measurements provided by PMUs, not processed by a state estimator.

\section{F. Analysis of marginal cases}

In order to check the reliability of the criterion, sensitivities have been tested in stringent marginal situations. To this purpose the system stress has been decreased by reducing the pre-disturbance load in the Central area in small steps, until the disturbance stops causing instability. The so obtained marginally stable case corresponds to a load decrease of 270 MW / 90 Mvar. By stepping back, a marginally unstable case has been created, corresponding to a load decrease of $240 \mathrm{MW}$ / 80 Mvar. The disturbance is the tripping of line 4032-4044 at $t=1 \mathrm{~s}$, without short-circuit this time.

We first consider the marginally unstable case. In this scenario, the system evolves very slowly under the effect of LTCs and OELs. The latter act on generators g7, g14, g15, g16, g12 and g6 at $t=86.8,93.1,496.3,663.8,699.3,802.3 \mathrm{~s}$, respectively. The system eventually collapses at $t \simeq 833 \mathrm{~s}$. Figure 10 shows the evolution of the voltage magnitude at the transmission bus already considered in Fig. 3 .

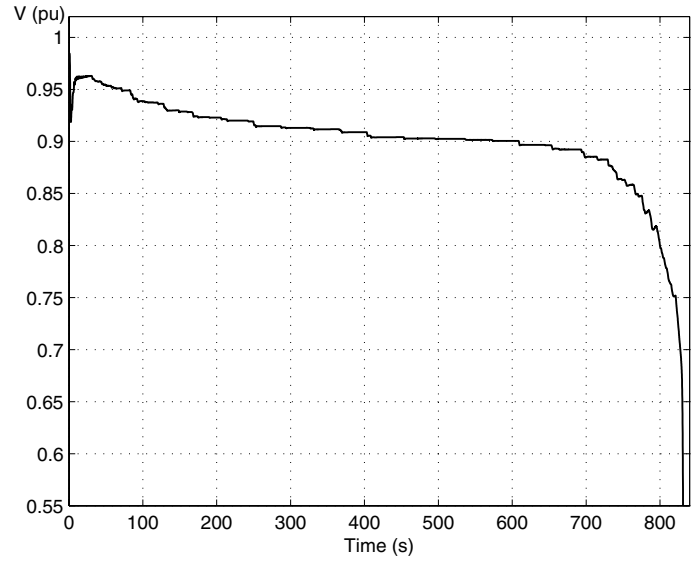

Fig. 10. Evolution of voltage at bus 1041 in marginally unstable case

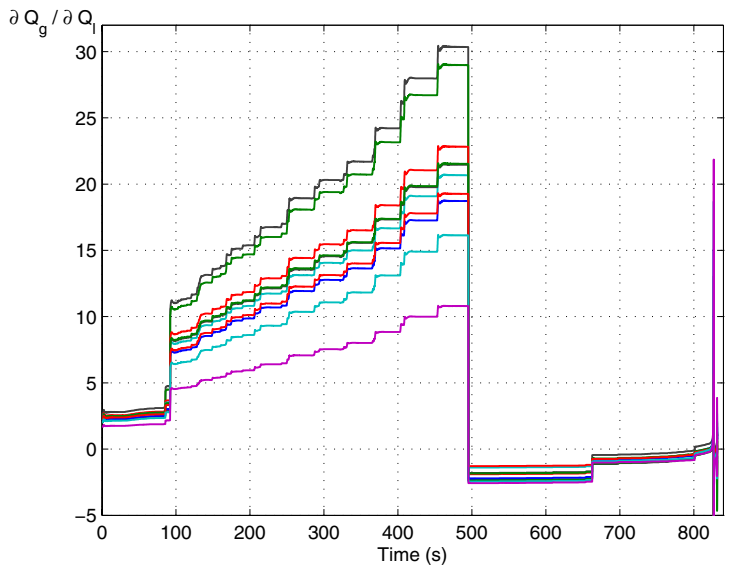

Fig. 11. Sensitivities $S_{Q_{g} \mathbf{Q}_{\mathbf{l}}}$ in marginally unstable case

The corresponding sensitivities at buses of the Central area are shown in Fig. 11. The change in the sign of the sensitivities happens at $t=495.2$ s, i.e. 494 seconds after the disturbance and 348 seconds before collapse, thus giving an early warning of the slowly coming instability, even in this marginally unacceptable situation.

The results in the marginally acceptable case are even more interesting. With the load decreased by 270 MW / 90 Mvar, the system response to the disturbance is the one shown in Fig. 12. The system reaches a new long-term equilibrium after a long time (almost $500 \mathrm{~s}$ ), as expected. In this case, OELs are activated on generators $\mathrm{g} 7$ at $t=100.2 \mathrm{~s}$ and $\mathrm{g} 14$ at $t=144.7 \mathrm{~s}$, thus leading to much fewer limitations.

The sensitivities at buses of the Central area are shown in Fig. 13. Although they reach large values indicating proximity to instability, they do not change sign. This means that no false alarm is issued in spite of the small security margin left to the system.

The proposed method thus shows promising in terms of dependability and security.

\section{CONCLUSION}

The problem of anticipatively detecting the onset of longterm voltage instability caused by a large disturbance has been addressed. The approach relies on the conjecture that in some future, it will be possible to make a voltage weak region fully 


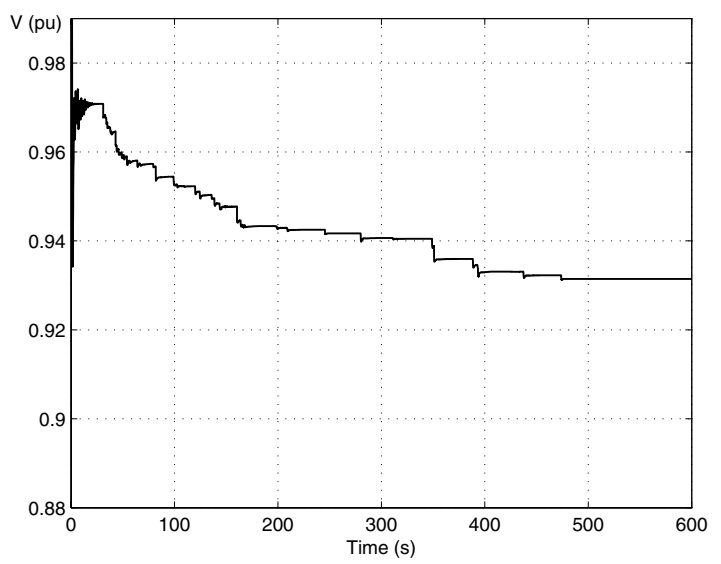

Fig. 12. Evolution of voltage at bus 1041 in marginally stable case

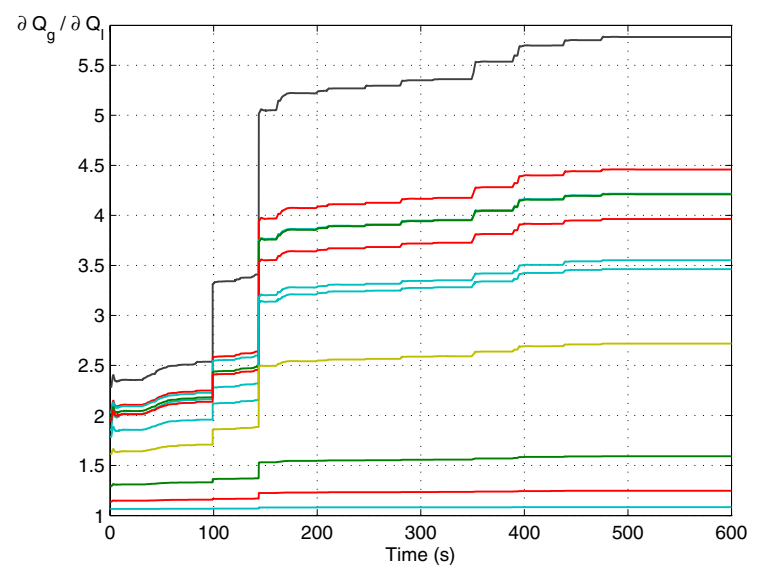

Fig. 13. Sensitivities $S_{Q_{g} \mathbf{Q}_{1}}$ in marginally stable case

observable through PMUs providing synchronized bus voltage measurements at a high rate.

An extended set of long-term equilibrium equations, taking into account the important effects of OELs and LTCs, is fitted to the system states provided by PMUs. Sensitivities are efficiently obtained from this real-time model, and their change in sign (through large values) is used as alarm criterion. No further tuning of alarm thresholds is needed. A moving average filter is incorporated to deal with the noise stemming inevitably from measuring devices and faster transients.

The initial results obtained from a test system are quite encouraging. They suggest that the proposed approach could make up the heart of a dependable and secure wide-area protection scheme. It has been also shown that filtering of the incoming voltage phasors should be preferred to filtering of sensitivities.

Work is underway to extend the proposed approach in several directions such as:

- quick identification of most efficient emergency controls

- further anticipation of near-future system evolution using fast simulation

- tests with faster restoring loads, in particular induction motors

- measurement processing through a (linear) state estimator

- use of more refined filters than MA

- method adaptation in case the region is partially observ- able through PMUs.

\section{REFERENCES}

[1] C. W. Taylor, Power System Voltage Stability, EPRI Power System Engineering Series, McGraw Hill, 1994

[2] T. Van Cutsem, C. Vournas, Voltage Stability of Electric Power Systems, Boston, Kluwer Academic Publishers, 1998

[3] P. Pourbeik, C. Rehtanz (Conveners), "Wide Area Monitoring and Control for Transmission Capability Enhancement, CIGRE Task Force 38.02.19, CIGRE Technical brochure, Jan. 2007

[4] C. Rehtanz, J. Bertsch, "Wide Area Measurement and Protection System for Emergency Voltage Stability Control", In Proc. of the IEEE PES Winter Meeting, pp. 842-847, 2002

[5] A. G. Phadke, "Synchronized Phasor Measurements in Power Systems", IEEE Computer Applications in Power, Vol. 6, No. 2, pp. 10-15, Apr. 1993

[6] R. F. Nuqui, A. G. Phadke, "Phasor Measurement Unit Placement Techniques for Complete and Incomplete Observability", IEEE Trans. on Power Delivery, Vol. 20, No. 4, pp. 2381-2388, Oct. 2005

[7] A. G. Phadke, J. S. Thorp, K. J. Karimi, "State Estimation with Phasor Measurements", IEEE Trans. on Power Systems, Vol. 1, No. 1, pp. 233241, Feb. 1986

[8] K. Vu, M. M. Begovic, D. Novosel, M. M. Saha, "Use of Local Measurements to Estimate Voltage Stability Margin", IEEE Trans. on Power Systems, Vol. 14, No. 3, pp. 1029-1035, Aug. 1999

[9] D. E. Julian, R. P. Schulz, K. T. Vu, W. H. Quaintance, N. B. Bhatt, D. Novosel, "Quantifying proximity to voltage collapse using the voltage instability predictor (VIP)", Proc. IEEE PES Summer Meeting, Seattle, Jul. 2000, pp. 16-20

[10] L. Warland, A. T. Holen, "Estimation of Distance to Voltage Collapse: Testing and Algorithm Based on Local Measurements", Proc. 14th Power System Computation Conference, Paper s38p03, Sevilla (Spain), Jun. 2002

[11] Zima M., Larsson M., Korba P., Rehtanz C., Andersson G., "Design aspects for wide-area monitoring and control systems", Proceedings of the IEEE, Vol. 93, No. 5, pp. 980-996, 2005

[12] I. Smon, G. Verbic, F. Gubina, "Local Voltage-Stability Index Using Tellegen's Theorem”, IEEE Trans. on Power Systems, Vol. 21, No. 3, pp. 1267-1275, Aug. 2006

[13] Y. Gong, N. Schulz, A. Guzman, "Synchrophasor-Based Real-Time Voltage Stability Index", Proc. Power System Conference and Exposition, Atlanta, Oct./Nov. 2006

[14] C. D. Vournas, N. G. Sakellaridis, "Tracking Maximum Loadability Conditions in Power Systems", Proc. Bulk Power System Dynamics and Control-VII, Paper 104, Charleston, Aug. 2007

[15] B. Milosevic, M. Begovic, "Voltage Stability Protection and Control using a Wide-Area Network of Phasor Measurements", IEEE Trans. on Power Systems, Vol. 18, No. 1, pp. 121-127, Feb. 2003

[16] C. Canizares (Editor/Coordinator), Voltage Stability Assessment: Concepts, Practices, and Tools, IEEE PES Power System Stability Subcommittee, ISBN 0780378695, 2002

[17] B. Genet, J-C. Maun, "Voltage Stability Monitoring using WideArea Measurement System", Proc. of IEEE PowerTech Conf., Lausanne (Switzerland), paper 392, Jun. 2007

[18] G. K. Morison, B. Gao, P. Kundur, "Voltage Stability Analysis Using Static and Dynamic Approaches", IEEE Trans. on Power Systems, Vol. 8, No. 3, pp. 1159-1171, Aug. 1993

[19] T. Van Cutsem, Y. Jacquemart, J.-N. Marquet, P. Pruvot, "A comprehensive analysis of mid-term voltage stability", IEEE Trans. on Power Systems, Vol. 10, No. 3, pp. 1173-1182, Aug. 1995

[20] F. Capitanescu, T. Van Cutsem, "Unified sensitivity analysis of unstable or low voltages caused by load increases or contingencies", IEEE Trans. on Power Systems, Vol. 20, No. 1, pp. 321-329, Feb. 2005

[21] V. Balamourougan, T. S. Sidhu, M. S. Sachdev, "Technique for online prediction of voltage collapse", IEE Proc. on Generation, Transmission and Distribution, Vol. 151, No. 4, pp. 453-460, Jul. 2004

[22] M. Kezunovic, "Monitoring of Power System Topology in Real-Time", In Proc. 39-th International Conference on System Sciences, Hawaii, Jan. 2006

[23] M. Stubbe (Convener), Long-Term Dynamics - Phase II, CIGRE TF 38.02.08, CIGRE Technical brochure, Jan. 1995

[24] I. Dobson, L. Lu, "Voltage Collapse Precipitated by the Immediate Change in Stability When Generator Reactive Power Limits are Encountered", IEEE Trans. on Circuits and Systems-1: Fundamental Theory and Applications, Vol. 39, No. 9, pp. 762-766, Sep. 1992 